\title{
Dengue, Guillain-Barré Syndrome, and Cerebral Infarction: A Case of Rare Complication
}

\author{
Prateek $^{1}$, Vandana Sharma ${ }^{2}$, Naveen Paliwal ${ }^{3}$, Himani Tak ${ }^{4}$
}

\begin{abstract}
Incidence of cerebral infarction after use of intravenous immunoglobulin (IVIG) for treatment of Guillain-Barré syndrome (GBS) is relatively uncommon. Here, we discuss a case of 30-year-old female who was admitted with a complain of thrombocytopenia after infection with dengue virus, who then developed GBS just after a day of discharge. But her woes did not end there, as the GBS progressed rapidly involving her respiratory muscles, leaving her in need of ventilation assistance. Her condition was further deteriorated by development of cerebral infarction that may have been precipitated by the administration of IVIG during the early course of management.

Keywords: Cerebral infarction, Dengue, Guillain-Barré syndrome, Intravenous immunoglobulin.

Key messages: Intravenous immunoglobulin in a GBS patient with a hematological abnormality (dengue) should be used with caution. Therapeutic plasma exchange may be considered for management in cases with variable coagulability.

Indian Journal of Critical Care Medicine (2019): 10.5005/jp-journals-10071-23285
\end{abstract}

\section{INTRODUCTION}

Dengue is a common mosquito-borne infection in India with manifestations ranging from flu-like illness to severe hemorrhagic complications, but this does not preclude the neurological complications, which range from $0.5 \%$ to $6.2 \%$. ${ }^{1}$ Guillain-Barré syndrome is an immune-mediated illness of peripheral nervous system with usual presentation of acute ascending flaccid paralysis, areflexia, and dysautonomia. Mild cases of GBS are usually managed with conservative and supportive measures, but rapidly progressive GBS involving respiratory muscles is usually treated with either IVIG or therapeutic plasma exchange (TPE), depending on the local preference and cost efficacy. Only a few reports have been published regarding thromboembolic complications of IVIG, since its use for treatment of GBS. ${ }^{2}$

Here, we are discussing one such case, where patient developed cerebral infarction after the administration of IVIG for the treatment of GBS, who was recuperating from acute dengue illness (Fig. 1).

\section{Case Description}

A 30-year-old female was admitted with complaints of weakness in lower limb since 1 day. She had been discharged from the hospital a day before, after recuperating from the acute episode of dengue, for which she had been hospitalized for 5 days. On the basis of symptoms, dengue fever was managed conservatively. Over that period, a unit of single-donor platelet was transfused due to severe thrombocytopenia. After patient's readmission, due to acute onset of weakness, which progressed to both lower and upper limbs in the following days, GBS was suspected. All the motor reflexes were absent. The patient was conscious, oriented to time, place, and person with no sign of neck rigidity or any alteration in sensorium, which also clinically favored the diagnosis of GBS. Cerebrospinal fluid (CSF) analysis revealed albuminocytological dissociation. As the weakness progressed, treatment was initiated for GBS with IVIG at a dose of $0.4 \mathrm{~g} / \mathrm{kg} /$ day for 5 days. Still, a noncontrast CT scan was carried out to exclude the recurrence of intracranial space occupying lesion (ICSOL), as the patient had been operated upon for a cystic
${ }^{1-3}$ Department of Anesthesiology and Critical Care, Dr Sampurnanand Medical College, Jodhpur, Rajasthan, India

${ }^{4}$ Department of Community Medicine, Dr Sampurnanand Medical College, Jodhpur, Rajasthan, India

Corresponding Author: Vandana Sharma, Department of Anesthesiology and Critical Care, Dr Sampurnanand Medical College, Jodhpur, Rajasthan, India, Phone: +91 9460157024, e-mail: neopatricks@outlook.com

How to cite this article: Prateek, Sharma V, Paliwal N, Tak H. Dengue, Guillain-Barré Syndrome, and Cerebral Infarction: A Case of Rare Complications. IJCCM 2019;23(11):533-535.

Source of support: Nil

Conflict of interest: None

cerebellar astrocytoma 15 years back, with ventriculo-peritoneal (VP) shunt left in situ. On 5th day of her admission, patient had to be intubated and ventilated mechanically due to development of respiratory failure. As there was not much clinical improvement in muscle power over few days, patient was tracheostomized on 5th ventilator day (i.e., 10th day of admission). Meanwhile, supportive treatment was being continued throughout this period including deep vein thrombosis (DVT) prophylaxis with low molecular weight heparin (LMWH). Dexmedetomidine infusion was started to keep the patient sedated for maintaining ventilation synchrony, with intermittent sedation holds for assessing clinical improvement.

Patient was showing signs of recovery, with slight improvement in muscle power to grade $2 / 5$ in both the upper limbs and $1 / 5$ in lower limbs. Suddenly, on 18th day of the admission, a diminished response of the patient was noticed, despite hemodynamic stability and without any episode of hypoxia or hypotension. MRI was planned accordingly, which revealed an acute cerebral infarct in the left parieto-occipital region. Prothrombin time, activated partial thromboplastin time, thrombin time, bleeding time, and clotting time were carried out to rule out any thrombophilic tendency (though prothrombin time/ international normalized ratio (PT/INR)

() The Author(s). 2019 Open Access This article is distributed under the terms of the Creative Commons Attribution 4.0 International License (https://creativecommons. org/licenses/by-nc/4.0/), which permits unrestricted use, distribution, and non-commercial reproduction in any medium, provided you give appropriate credit to the original author(s) and the source, provide a link to the Creative Commons license, and indicate if changes were made. The Creative Commons Public Domain Dedication waiver (http://creativecommons.org/publicdomain/zero/1.0/) applies to the data made available in this article, unless otherwise stated. 


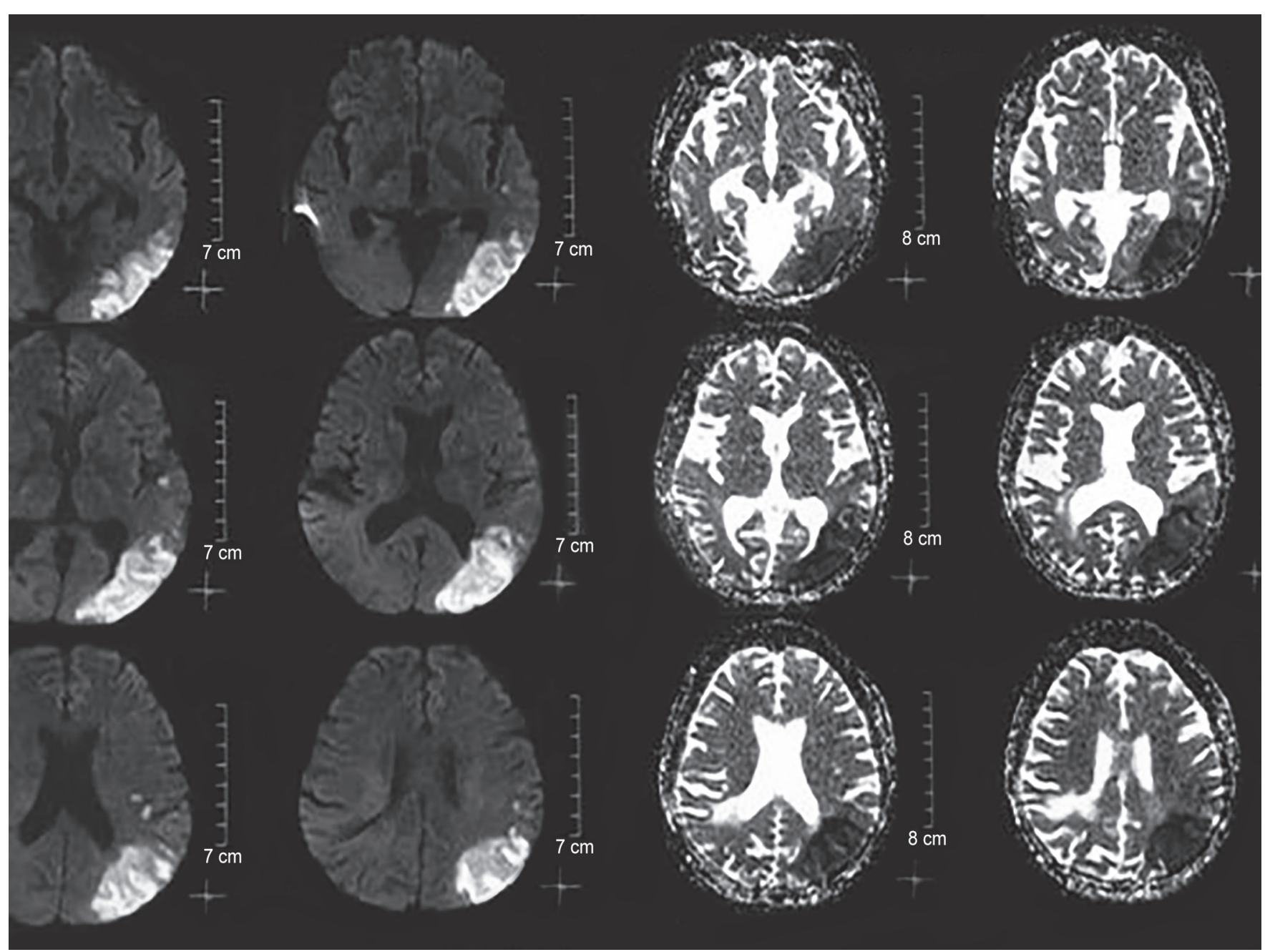

Fig. 1: Cerebral infarct in the dengue patient

had already been done, before carrying out tracheostomy) and were found to be normal. Lipid prolife and 2D echo were also normal. After the incidence of cerebral infarct, the patient also developed focal seizures of facial muscles, which had to be treated with a combination of antiepileptics, including sodium valproate and levetiracetam. In the following days, patient's treatment continued on the basis of new symptoms, with specific focus on hemodynamic management and sepsis control. The patient's condition improved over a period of 3 months. She was shifted out from ICU after decannulation of the trachea, when she was no longer in need of the ventilator support. At the time of discharge, patient had a modified Rankin score of $4 .^{3}$

\section{Discussion}

Cerebral infarction can be caused by multitude of factors, which include both modifiable and nonmodifiable factors, but the basis for thrombosis always remains the same that is summarized by Virchow's triad as hypercoagulability, stasis, and endothelial injury. ${ }^{4,5}$ The case in discussion had various factors that could have led to cerebral infarction, which are being discussed in detail here.

Dengue is a common mosquito-borne (Aedes mosquito) infection in tropical countries especially India. Its classical symptoms include flu-like illness, retro-orbital pain, maculopapular rash, petechiae that may be complicated by dengue hemorrhagic fever, and dengue shock syndrome. Dengue is a nonneurotropic virus but it may cause neurological symptoms including GBS and even thromboembolic phenomenon like ischemic stroke. Pathology behind GBS remains unclear and is perceived to be immunemediated while ischemic stroke is considered to be caused by meningovasculitis or a transient hypercoagulable state. ${ }^{6}$

Guillain-Barré syndrome is an immune-mediated disorder of peripheral nervous system with classical symptoms of acute flaccid paralysis, areflexia, and dysautonomia. It is usually managed with supportive measure unless rapidly progressing, when specific management with IVIG or TPE needs to be carried out. Intravenous immunoglobulin is recommended early in severe GBS as the treatment option, with the dosage of $0.4 \mathrm{~g} / \mathrm{kg} /$ day for 5 days. Intravenous immunoglobulin is reported to have severe complications ranging from nausea and fever to acute renal failure and anaphylaxis (Table 1). ${ }^{7}$ Rarely, cases of cerebral infarction have been reported after management of GBS with IVIG, though patients in question usually had vascular risk factors. ${ }^{8}$ Cerebral infarction is thought to be secondary to hyperviscosity, thromboemboli, vasculitis, or cerebral vasospasm. ${ }^{2}$

Dysautonomia due to GBS results in wide fluctuations of hemodynamic parameters, i.e., brady/tachycardia and hypo/ hypertension; combined with the hypercoagulability caused by the use of IVIG may have caused such a cerebral infarct of watershed areas in this patient, as the patient had no other risk factors for thrombophilia. 


\begin{tabular}{ll} 
Table 1: Adverse effects of intravenous immunoglobulin & \\
\hline Frequency & Adverse effect \\
\hline Common & Fever \\
& Myalgia \\
& Nausea \\
& Shaking \\
& Headache \\
& Hypotension \\
Uncommon & Urticaria \\
& Eczema \\
& Leukopenia \\
Rare & Acute renal failure \\
& Aseptic meningitis \\
& Cerebral infarction \\
Very rare & Myocardial infarction \\
\end{tabular}

Therapeutic plasma exchange is an equally effective option for treatment of GBS in early course of treatment. It is usually administered as $50 \mathrm{~mL} / \mathrm{kg}$ on five separate occasions over 2 weeks. Major hemostatic disorders, unstable cardiovascular state, and active infection are contraindications to $P E .{ }^{9}$ This patient could have been better served with use of TPE with plasma as a fluid of choice for exchange, as albumin use leads to transient fall in clotting factors which can cause increased incidence of bleeding.

\section{Conclusion}

Intravenous immunoglobulin and TPE both are good treatment options for GBS but use of TPE could be preferred in such cases where initial disease (e.g., dengue) may have been associated with hematovascular complications.

\section{References}

1. Murthy J. Neurological complications of dengue infection. Neurol India 2010;58(4):581-584. DOI: 10.4103/0028-3886. 68654.

2. Chang T, de Alwis JS, Samarasekara N, Rajapakse S. Cerebral infarction 3 weeks after intravenous immunoglobulin for miller fisher syndrome: a case report. J Med Case Rep 2014;8:100. DOI: 10.1186/1752-1947-8-100.

3. Wilson JT, Hareendran A, Grant M, Baird T, Schulz UG, Muir KW, et al. Improving the assessment of outcomes in stroke: use of a structured interview to assign grades on the modified Rankin scale. Stroke 2002;33(9):2243-2246. DOI: 10.1161/01.STR.0000027437. 22450.BD.

4. Goldstein LB, Bushnell CD, Adams RJ, Appel LJ, Braun LT, Chaturvedi S, et al. Guidelines for the primary prevention of stroke: a guideline for healthcare professionals from the American Heart Association/ American Stroke Association. Stroke 2011;42(2):517-584. DOI: 10.1161/ STR.0b013e3181fcb238.

5. DeWalt DA, Pincus T. The legacies of Rudolf Virchow: cellular medicine in the 20th century and social medicine in the 21st century. Isr Med Assoc J 2003;5(6):395-397.

6. Liou LM, Lan SH, Lai CL. Dengue fever with ischemic stroke: a case report. Neurologist 2008;14(1):40-42. DOI: 10.1097/NRL. Ob013e3180d0a391.

7. Pritchard J, Hughes RA. Intravenous immunoglobulin-how to use it practical. Neurology 2001;1:93-97.

8. Katz U, Shoenfeld Y. Review: intravenous immunoglobulin therapy and thromboembolic complications. Lupus 2005;14(10):802-808. DOI: 10.1191/0961203303lu2168rr.

9. Meena AK, Khadilkar SV, Murthy JMK. Treatment guidelines for Guillain-Barré syndrome. Ann Ind Acad Neurol 2011;14(Suppl 1): 73-81. DOI: 10.4103/0972-2327.83087. 